\title{
The host STING pathway at the interface of cancer and immunity
}

\author{
Leticia Corrales, ${ }^{1}$ Sarah M. McWhirter, ${ }^{2}$ Thomas W. Dubensky Jr., ${ }^{2}$ and Thomas F. Gajewski ${ }^{1,3}$ \\ 'Department of Pathology, The University of Chicago, Chicago, Illinois, USA. ${ }^{2}$ Aduro Biotech, Berkeley, California, USA. ${ }^{3}$ Department of Medicine, Section of Hematology/Oncology, \\ The University of Chicago, Chicago, Illinois, USA.
}

\begin{abstract}
A major subset of human cancers shows evidence for spontaneous adaptive immunity, which is reflected by the presence of infiltrating $\mathrm{CD8}^{+} \mathrm{T}$ cells specific for tumor antigens within the tumor microenvironment. This observation has raised the question of which innate immune sensing pathway might detect the presence of cancer and lead to a natural adaptive antitumor immune response in the absence of exogenous infectious pathogens. Evidence for a critical functional role for type I IFNs led to interrogation of candidate innate immune sensing pathways that might be triggered by tumor presence and induce type I IFN production. Such analyses have revealed a major role for the stimulator of IFN genes pathway (STING pathway), which senses cytosolic tumor-derived DNA within the cytosol of tumor-infiltrating DCs. Activation of this pathway is correlated with IFN- $\beta$ production and induction of antitumor T cells. Based on the biology of this natural immune response, pharmacologic agonists of the STING pathway are being developed to augment and optimize STING activation as a cancer therapy. Intratumoral administration of STING agonists results in remarkable therapeutic activity in mouse models, and STING agonists are being carried forward into phase I clinical testing.
\end{abstract}

\section{Introduction}

The observation of spontaneous $\mathrm{T}$ cell responses against tumors suggests that endogenous adjuvants might provide signals for activation of antigen presenting cells (APCs). Pattern recognition receptors (PRRs) expressed by APCs typically recognize molecular entities derived from infectious agents, which trigger innate immune responses but also lead to APC activation and induction of adaptive T cell responses. Multiple families of PRRs have been identified that reside in the plasma membrane, within intracellular vesicles, and in the cytosol of APCs. These include TLRs (1), C-type lectin receptors (CLRs) (2), NOD-like receptors (NLRs) (3), retinoic-inducible gene-1-like (RIG-I-like) receptors (RLRs) (4), and cytosolic DNA sensors (5). Binding of ligands to PRRs activates adaptor molecules and downstream signaling events, leading to the secretion of type I IFNs, inflammatory cytokines, chemokines, and antimicrobial peptides. These factors orchestrate innate immune responses that initiate pathogen clearance but also result in maturation of APCs (in particular DCs), which in turn prime and activate antigen-specific $\mathrm{T}$ cells.

In the tumor setting, recent evidence has indicated that the major innate immune pathway involved in the generation of a spontaneous antitumor $\mathrm{T}$ cell response is the stimulator of IFN genes (STING) pathway of cytosolic DNA sensing $(6,7)$. Based on this finding, deliberate activation of the STING pathway has been explored as a cancer therapy, and STING agonists have been found to induce profound tumor control via host immune cell activation (8). In this

Conflict of interest: S.M. McWhirter and T.W. Dubensky Jr. are paid employees of Aduro Biotech. T.F. Gajewski and L. Corrales have a patent application pending through the University of Chicago for the use of STING agonists as a cancer therapeutic (PCT/ US14/66436).

Reference information: J Clin Invest. 2016;126(7):2404-2411. doi:10.1172/JCI86892. review, we will summarize the most recent literature on the molecular basis of the activation of the endogenous STING pathway in the recognition of tumors, as well as the rationale and progress in the development of STING agonists as tumor immunotherapies.

\section{Type I IFNs in the generation of antitumor immune responses}

Evaluation of gene expression profiles of melanoma metastases revealed two major tumor microenvironment phenotypes: a $\mathrm{T}$ cell-inflamed phenotype characterized by the presence of $\mathrm{T}$ cell markers and chemokines related to $\mathrm{T}$ cell recruitment (in particular CXCL9 and CXCL10) (9-11) and a non-T cell-inflamed phenotype that lacks expression of these immune-related genes. The $\mathrm{T}$ cell-inflamed phenotype is characterized by high levels of immune inhibitory factors, including programmed death-ligand 1 (PD-L1), the tryptophan-catabolizing enzyme indoleamine 2,3-dioxygenase (IDO), and FOXP3 ${ }^{+}$Tregs $(10,12)$, which likely are indicative of tumor escape from a successfully activated antitumor immune response. Importantly, clinical data have revealed that patients with this T cell-inflamed tumor phenotype are the most likely to respond to several immunotherapies, including therapeutic cancer vaccines (9), high-dose IL-2 (13), and antibodies against the inhibitory receptors cytotoxic T-lymphocyte associated-4 (CTLA-4) (14, 15) and PD-1 (16). Preclinical studies and translational biomarker analyses have suggested that the therapeutic activity of these immunotherapies is associated with reactivation of $\mathrm{T}$ cells already within the tumor microenvironment $(17,18)$. Similarly, the presence of activated $\mathrm{CD}^{+} \mathrm{T}$ cells in solid tumors correlates with better prognosis in patients with several different malignancies (19-23).

While it is conceivable that tumor-infiltrating lymphocytes (TILs) might be a nonspecific reflection of inflammation, several lines of evidence suggest that at least a subset of these $\mathrm{T}$ cells can 


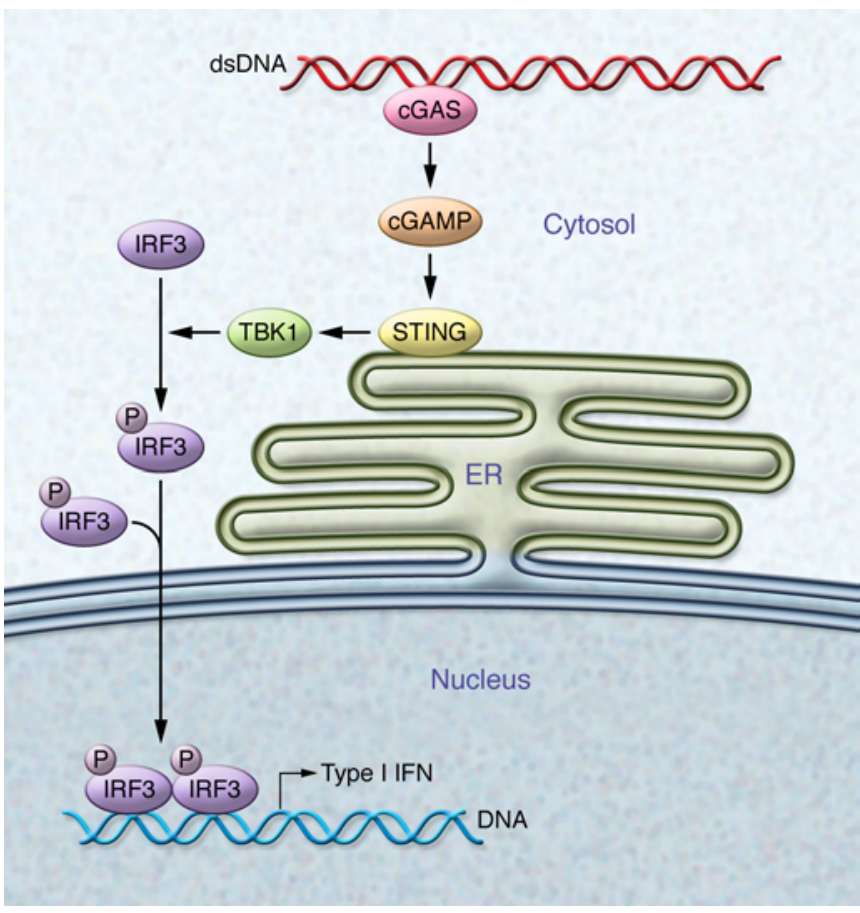

recognize tumor antigens. CD8 ${ }^{+}$TILs that are specific for differentiation antigens such as MelanA (MART-1) have been identified in melanoma (24). Moreover, TIL-based adoptive T cell therapy as developed by the Rosenberg group, which generates clinical responses in excess of $50 \%$ in metastatic melanoma patients, uses $\mathrm{T}$ cells from the tumor microenvironment as a starting point, indicating that tumor antigen-specific $\mathrm{T}$ cells must reside there, even if they are maintained in a dysfunctional state in situ. Recent work to molecularly identify tumor antigens associated with clinical response in such patients has revealed that many TILs are specific for mutated antigens generated as a consequence of somatic genomic instability (25). With checkpoint blockade therapy, expansion of circulating $\mathrm{T}$ cells against specific antigens has been observed following treatment (26), and oligoclonality of TILs following anti-PD-1 mAb treatment also has been reported (18). As such, there is substantial evidence that an antigen-specific $\mathrm{T}$ cell response becomes generated in a major subset of cancer patients. However, until recently, the mechanism by which this adaptive immune response becomes primed in the absence of the innate immune-activating molecular patterns typically provided by components of exogenous infectious pathogens had remained elusive.

The first clue that innate immune pathways might be activated in the tumor context came from the analysis of gene expression profiling of melanoma metastases. It was observed that the presence of T cell-associated transcripts correlated with expression of genes known to be induced by type I IFNs (10). The type I IFN signature, similarly to $\mathrm{T}$ cell infiltration itself, has been reported to predict favorable clinical outcome to therapeutic cancer vaccines (11), as well as to anthracycline-based chemotherapy in patients with breast carcinoma (27). Type I IFNs can act at several levels within the generation of an adaptive $\mathrm{T}$ cell response, promoting cross-priming of antigens by APCs and their migration to lymph nodes, thereby enhancing the effector functions of CTLs and
Figure 1. Scheme showing the activation of the STING pathway by cytosolic DNA. Cytosolic DNA is recognized by cGAS, which catalyzes the generation of CGAMP. cGAMP binds to STING and leads to its activation, which involves translocation from the ER to perinuclear sites. This translocation results in the recruitment and activation of TBK1 by autophosphorylation. Active TBK1, in turn, phosphorylates the transcription factor IRF3, which translocates to the nucleus to induce transcription of type I IFN genes.

supporting the survival of memory CTLs (28). Importantly, in the tumor setting, signaling through the type I IFN receptor on host hematopoietic cells has been shown to be critical for the generation of an immunologic response against chemically induced sarcomas (29). In mouse transplantable tumor models, host type I IFN signaling within APCs was required for spontaneous regression of immunogenic tumors $(6,30)$. In fact, type I IFN signaling was required specifically within a minor subset of DCs identified by the transcription factor BATF3. Murine BATF3-lineage DCs express the markers CD8 $\alpha$ or CD103 and are the most potent type of APC for presentation of antigens to CD8 ${ }^{+} \mathrm{T}$ cells. (31). In addition, signaling through host type I IFN receptor (IFNAR) was shown to regulate bone metastasis of breast cancer cells (32). Spine metastases showed diminished expression of more than 500 IFN-regulated genes (IRGs) compared with the primary tumors, suggesting that expression of IRGs protects against metastases (32). Thus, similarly to most viral infections, host type I IFN signaling appears to be crucial for inducing an adaptive immune response against tumors.

Apart from boosting antitumor immune responses, type I IFNs have been implicated in tumor suppression through the induction of apoptosis and antiproliferative responses acting directly on tumor cells (33). Therefore, it is reasonable to consider that tumor cells might attenuate production of type I IFNs in order to escape from these tumor-regulatory consequences. Indeed, most immortalized and tumor cell lines, but not primary cells, fail to generate type I IFNs in response to intracellular DNA (34). One of the molecular mechanisms that may explain this observation is that certain oncoproteins antagonize innate immune signaling that leads to the transcription of type I IFNs and other immune cytokines $(35,36)$. Another mechanism that has been observed in both leukemia and glioma cells is the homozygous deletion of type I IFN genes $(37,38)$. Consistent with these data, it has been demonstrated that the main cell types that produce IFN- $\beta$ within the tumor microenvironment are innate immune cells (predominantly DCs) and endothelial cells $(6,39)$.

A critical question was the identity of the major innate immune pathway that detects the presence of tumor cells and/or tumorderived factors in order to trigger the production of type I IFNs by host APCs. Mechanistic studies using mouse transplantable tumor models showed that signaling through TLRs, the cytosolic RNA sensing pathway mediated by mitochondrial antiviral-signaling protein (MAVS), or the purinergic receptor P2X ligand-gated ion channel 7 (PX72R) were dispensable in the generation of spontaneous $\mathrm{T}$ cell priming against tumor antigens. However, deficiency in STING (also known as transmembrane protein 173 [TMEM173], MITA, ERIS, and MPYS) or the transcription factor IFN regulatory factor 3 (IRF3) resulted in markedly diminished host IFN- $\beta$ production and $\mathrm{T}$ cell priming, leading to defective growth control 
of immunogenic tumors (7). A large fraction of tumor-infiltrating DCs showed the presence of tumor-derived DNA in the cytosol that correlated with IRF3 translocation to the nucleus and expression of IFN- $\beta$. Together, these data suggest that the main innate immune sensing pathway activated within APCs in the context of a growing tumor is the STING pathway.

\section{Regulation of the STING pathway in APCs and its role in cancer}

STING is an adaptor protein anchored in the ER. In its basal state, STING exists as a dimer, with its C-terminal domain residing in the cytosol; however, in the presence of cytosolic DNA - typically due to viral, bacterial, or parasitic infections (5), but also under conditions in which mammalian DNA itself can gain access to the cytosol $(40,41)$ - STING undergoes conformational changes and transits from the ER through the Golgi to perinuclear endosomes by a mechanism that seems to require components of the autophagosome (42). Consequently, STING recruits TANK-binding kinase 1 (TBK1), which phosphorylates STING, rendering it more accessible for the binding of IRF3 (43). TBK1 then phosphorylates IRF3, which translocates to the nucleus to drive transcription of IFN- $\beta$ and other innate immune genes (refs. 44-46 and Figure 1).

Since 2009, several putative cytosolic DNA receptors have been explored for the potential to drive STING activation (47); however, none of these receptors was universally accepted to be the major DNA receptor for activation of this pathway. The discovery of the bacterial second messengers cyclic-dinucleotides (CDNs) $(48)$ as natural ligands of STING $(44,49)$ provided clues regarding the endogenous mechanism that links the presence of cytosolic DNA to the activation of STING. Importantly, it was found that, not only bacteria, but also mammalian cells generate CDNs (50-53) via the DNA sensor cyclic GMP-AMP synthase (cGAS/MB21D1), which catalyzes the synthesis of cyclic GMPAMP (cGAMP) from GTP and ATP upon DNA binding (54). Cells from cGAS-deficient mice were unable to produce type I IFNs in response to cytosolic DNA (55). Overall, it is now generally accepted that upon DNA exposure within the cytosol, cGAS is the major receptor that directly binds DNA, leading to cGAMP production, which in turn engages STING to trigger the remaining signaling events that drive IFN- $\beta$ expression.

STING pathway activation within APCs in the tumor microenvironment leads to production of IFN- $\beta$ and the spontaneous generation of antitumor $\mathrm{CD}^{+} \mathrm{T}$ cell responses, allowing for control of the growth of several transplantable tumor cell models (7). Although the STING pathway is critical for $\mathrm{T}$ cell priming and $\mathrm{T}$ cell-based rejection in the tumor context, it is not required for general rejection of all types of tissues. Using a skin graft rejection model across minor histocompatibility antigen differences (56), the rate of graft rejection when STING-deficient male skin was transplanted into STING-deficient female recipients was identical to the rate of rejection in WT donor-recipient pairs (7). Thus, not all tissue-based T cell rejection processes are STING dependent.

The protective role of STING signaling in tumorigenesis has been confirmed using additional in vivo model systems. In a model of colitis-associated carcinogenesis (CAC) induced by azoxymethane/dextran sodium sulfate (AOM/DSS), STING deficiency increased the susceptibility to both colitis and tumor formation (57, 58). Mechanistically, AOM induced DNA damage and triggered STING signaling that activated inflammatory wound repair-initiating cytokines, such as IL-1 $\beta$ and IL-18, and suppressed growth inhibitory IL-22 binding protein (IL-22BP) $(57,59,60)$. Colons from animals with dysfunctional STING showed augmented expression of the proinflammatory cytokines IL-6 and keratinocyte chemoattractant (KC), which correlated with increased phosphorylated ERK and phosphorylated I $\mathrm{B} \alpha$, along with active STAT3 (41). Persistent activation of STAT3 was associated with amplified inflammation and promotion of colon tumorigenesis (61).

In an inducible model of glioma generated using a sleeping beauty transposon system, animals with a mutation rendering STING nonfunctional (I199N) (62) produced lower levels of type I IFNs, mainly generated by brain-infiltrating CD11b ${ }^{+}$ cells, which correlated with reduced immune-mediated tumor control (63). In transplantable models of melanoma and lymphoma, activation of the STING pathway in CD11 $\mathrm{c}^{+}$cells after cryoablation has been reported to lead to the production of type I IFNs and generation of an adaptive immune response against tumor-associated antigens (64).

In contrast to the studies described above, activation of the STING pathway has also been linked to promotion of tumorigenesis in some model systems. STING deficiency has been reported to protect against cancer development in a model of cutaneous skin tumors induced by 7,12-dimethylbenz[a] anthracene (DMBA) (65). In this model, DNA released into the cytosol of carcinogen-damaged cells stimulated the STING pathway, driving the recruitment of phagocytes that in turn drove a process of inflammation that promoted tumor development $(65,66)$. DNA sensing by the STING pathway can also mediate immune regulatory responses by induction of IFN-stimulated genes (ISG) with immunoregulatory functions like IDO (67). It has been shown that stimulation of the STING pathway in myeloid DCs using DNA nanoparticles induces IDO, which activate Tregs to promote dominant inhibitory $\mathrm{T}$ cell regulation $(68,69)$. These data suggest that activation of the STING pathway can induce immune suppressive factors in the tumor microenvironment; thus, an appropriate level of STING pathway activation may be required for optimal antitumor effects.

\section{STING pathway activation in other cell types}

While activation of the STING pathway has been well characterized in APCs, its role in other cell types has been less studied. Within the tumor microenvironment, $\mathrm{T}$ cells, endothelial cells, and fibroblasts all have been observed to produce type I IFNs upon stimulation with STING agonists ex vivo; however, the magnitude of type I IFN production was substantially lower compared with macrophages and DCs (8). Treatment of T cells with STING agonists has been shown to upregulate expression of genes associated with cytotoxic $\mathrm{T}$ cell function, suggesting a potential $\mathrm{T}$ cell-intrinsic role of the STING pathway (70).

It appears that some tumor cells have evolved mechanisms to shut down activation of the STING pathway or generation of STING-dependent cytokines. A recent study using human colon cancer cell lines showed a defect in STING-mediated innate immune signaling, which in some cases was mediated by epigenetic silencing of STING or cGAS gene expression (71), a feature 

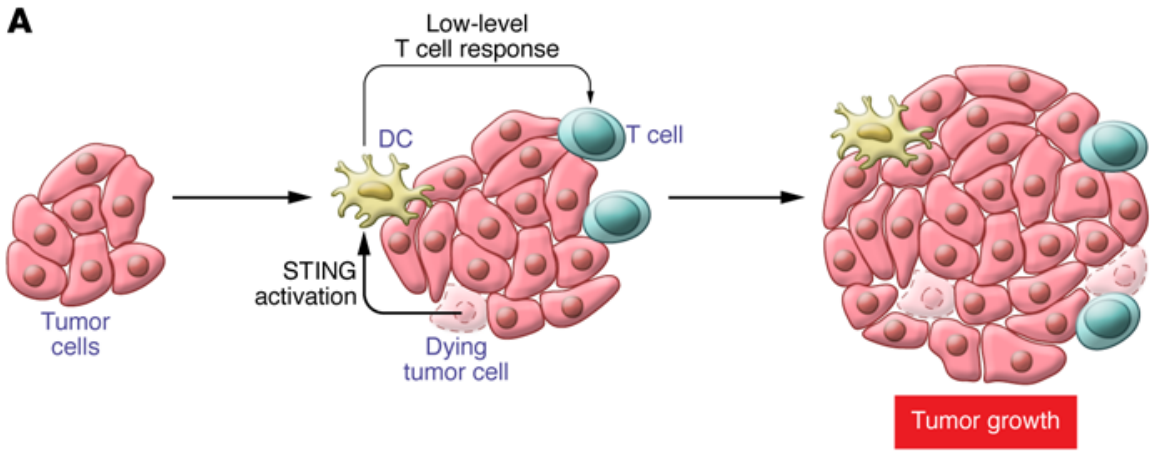

B

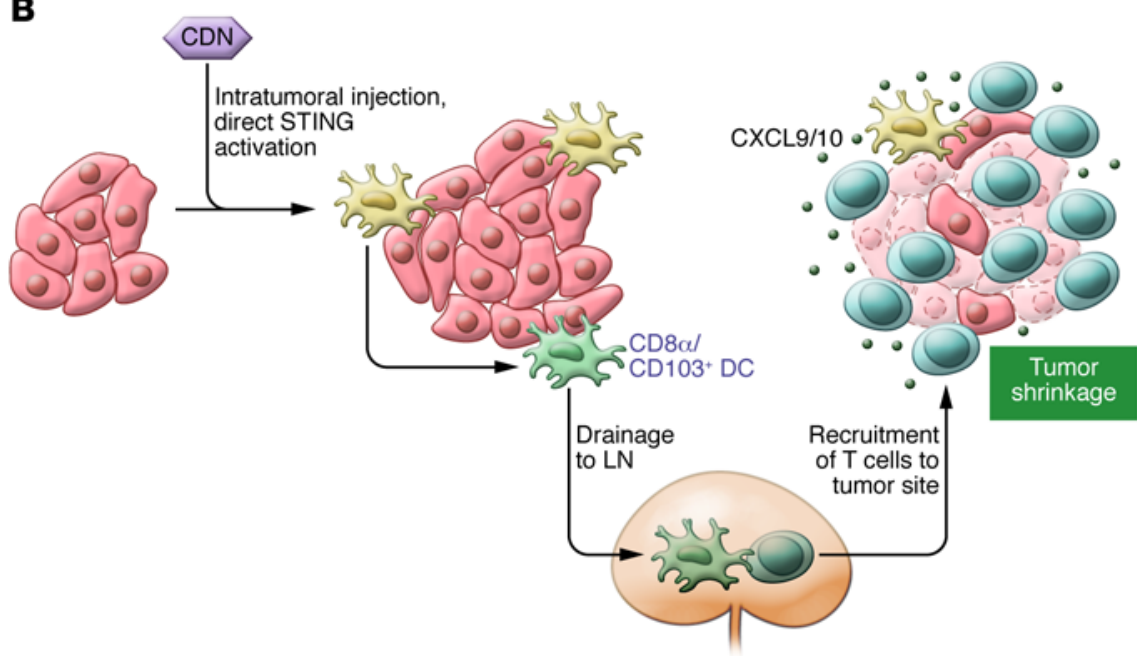

Figure 2. The STING pathway is activated within intratumoral DCs and boosts an adaptive immune response against tumors.

(A) Activation of STING in DCs by endogenous sources, most probably DNA released by dying tumor cells. DC activation via STING primes a low-level, endogenous $T$ cell response that is insufficient to overcome the immunosupressive tumor microenvironment. Thus, tumor growth continues in spite of activation of STING in DCs. (B) Intratumoral injection of synthetic STING agonists directly stimulates the STING pathway. Synchronized activation of DCs in the tumor leads to markedly augmented priming of tumor-specific T cells in tumor-draining lymph nodes (LNs). Downstream, recruitment of effector T cells into the tumor microenvironment is facilitated by the release of the chemokines CXCL9/10 from DCs and other cells at the tumor site. Successfully recruited antigen-specific effector T cells promote tumor cell killing, leading to measurable tumor shrinkage (8). that is shared by other tumor cell types (54). E7 and E1A oncoproteins expressed by the human papillomavirus (HPV) and adenoviruses, respectively, potently inhibit the cGAS-STING pathway by binding STING and blocking its activation (35). Thus, inhibition of STING pathway activation may represent a mechanism of immune evasion during carcinogenesis.

The STING pathway can become activated under some circumstances in which there is DNA damage. Exposure to $\gamma$-radiation or chemotherapeutic drugs such as etoposide leads to the release of DNA into the cytosol and stimulation of the STING pathway in APCs (72). The therapeutic effect of stereotactic radiation in vivo appears to depend on host STING, as well as type I IFNs and the resulting downstream T cell response (73). Together, these data indicate that the therapeutic effect of radiation is partially mediated by STING pathway activation. Further investigation will be needed to clarify whether chemotherapeutic agents that cause DNA damage also exert their therapeutic effect by stimulating the STING pathway in vivo, consequently promoting adaptive immune responses against tumors.

\section{Pharmacological use of STING agonists}

Based on the collective evidence indicating a role for endogenous STING pathway signaling in the generation of spontaneous immune responses against tumors, it was of interest to evaluate whether direct pharmacologic stimulation of the STING pathway might induce potent antitumor immunity and support rejection of poorly immunogenic tumors in vivo. It became possible to test the effects of STING activation when it was discovered that the well-characterized vascular disrupting agent 5,6-dimethyllxanthenone-4-acetic acid (DMXAA) (74) was in fact a direct agonist of mouse STING (75-78). DMXAA was previously known for its antivascular properties derived from a direct effect on tumor endothelial cells (79) or an indirect effect on macrophages as result of the induction TNF- $\alpha$ and NO generation by tumor-associated macrophages (TAM) $(80,81)$. DMXAA also induced repolarization of M2-like macrophages to an M1-like phenotype in a mouse model of non-small cell lung carcinoma (NSCLC) (82); a similar activity conferred by treatment with a synthetic CDN was recently shown (83). Armed with the knowledge that DMXAA interacted with mouse STING, in vivo experiments were reapproached from the perspective of DMXAA being a STING agonist. A single intratumoral injection of DMXAA was sufficient to promote rejection of B16 melanoma in most of the treated mice and was associated with a marked increase in the frequency of tumor-specific $\mathrm{CD}^{+} \mathrm{T}$ cells (8). The therapeutic effect of DMXAA was completely dependent on host STING, and $\mathrm{CD}^{+} \mathrm{T}$ cells were required for maximal tumor control. Interestingly, mice that completely rejected tumors were protected against a second challenge, and treatment of one tumor generated a therapeutic effect against a second distant tumor (8). This potent antitumor effect of DMXAA was also observed in transplantable mouse models of prostate cancer, breast cancer, and fibrosarcoma $(8,74)$.

Although DMXAA appears to be a potent agonist for mouse STING, it does not interact with or activate human STING due to distinct amino acid residues in human STING that render it unable to bind DMXAA (75-78). This discrepancy likely explains 
Table 1. Clinical trials utilizing intratumoral delivery of innate immune system agonists

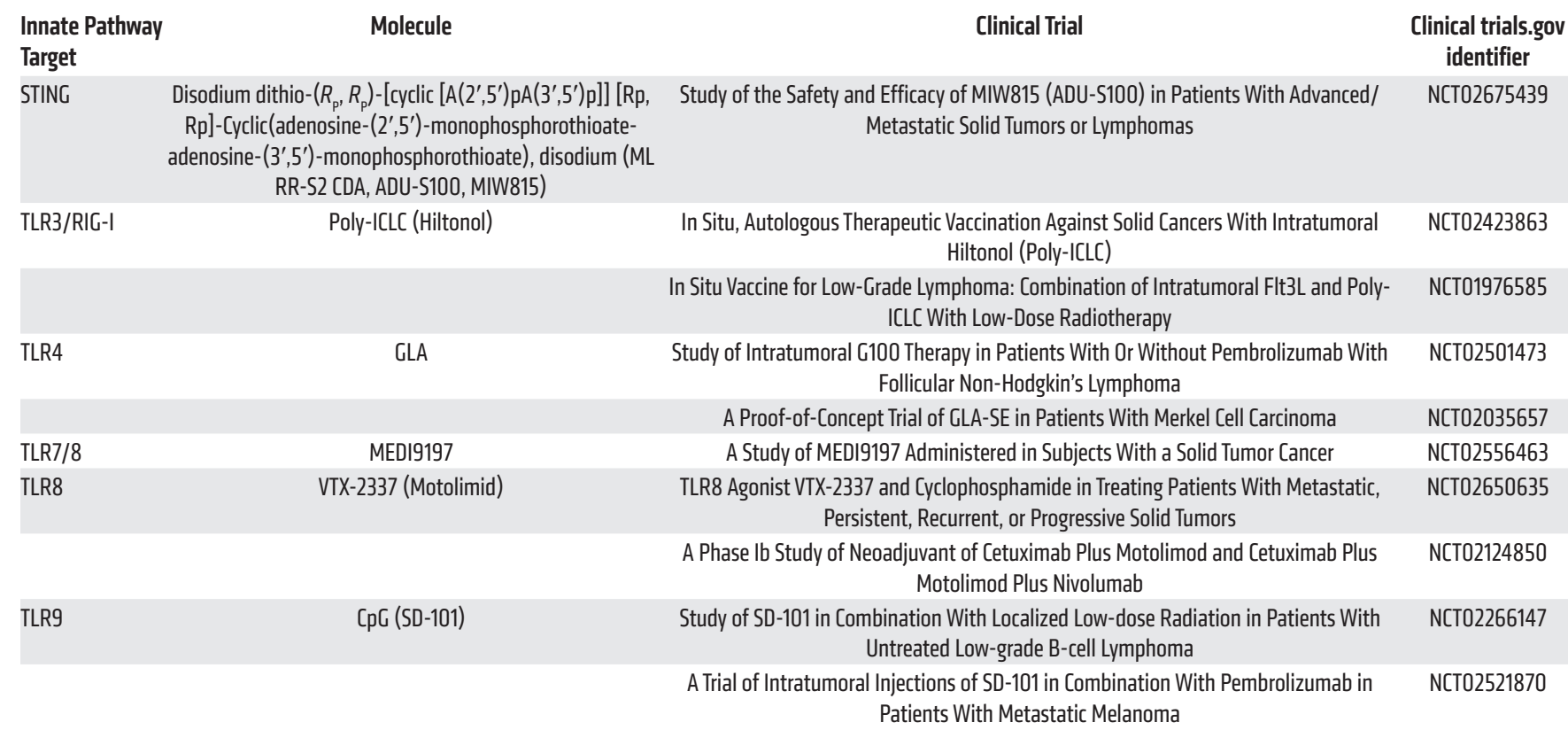

the negative results of the phase III clinical trial of DMXAA in patients with NSCLC (84). These recent insights have motivated a renewed search for effective agonists of human STING as a potential new approach for cancer immunotherapy.

\section{Development of human STING agonists as cancer immunotherapeutics}

Human STING agonists based on CDN ligands are being developed for clinical translation. STING-activating CDNs may have high translational potential as oncology therapeutics due to their potent antitumor activity as a single agent in aggressive mouse syngeneic tumor models $(8,63,83-86)$. Therapeutic antitumor efficacy with CDN-based STING agonists was initially demonstrated with daily i.p. injection of cyclic diguanylate (c-di-GMP) in the 4T1 mammary carcinoma model (85). However, c-di-GMP and other bacterial CDNs that have a canonical structure defined by a phosphate bridge with two $3^{\prime}-5^{\prime}$ linkages to join the two purine nucleotides into a cyclic molecule $\left(3^{\prime}, 3^{\prime} \mathrm{CDNs}\right)$ likely have limited translational value due to an inability to activate all human STING alleles D $(51,87)$. An analysis of the 1000 Genome Project database (http://www.1000genomes.org/) identified five human STING variants including the WT allele, the reference (REF) allele (R232H), the HAQ allele (R71H, G230A, R293Q), the AQ allele (G230A, R293Q), and the Q allele (R293Q) (87). A rationally designed synthetic CDN agonist, ML RR-S2 CDA, has been developed and exhibits enhanced stability, human STING activation, cellular uptake, and antitumor efficacy, as well as low reactogenicity compared with the natural STING ligands produced by bacteria or host cell cGAS $(8,86) . R_{\mathrm{p}}, R_{\mathrm{p}}(R, R)$ dithio-substituted diastereomer CDNs were resistant to digestion with phosphodiesterase, stimulated higher expression of IFN- $\beta$ in cultured human cells, and induced more potent antitumor immunity compared with CDNs that did not contain a dithio modification $(8,86)$. To increase affinity for human STING, ML RR-S2 CDA contains a noncanonical structure defined by a phosphate bridge with one $2^{\prime}-5^{\prime}$ and one $3^{\prime}-5^{\prime}$ mixed phosphodiester linkages (2',3' CDNs). The $2^{\prime}, 3^{\prime}$ mixed linkage structure confers increased STING binding affinity (52) and is also found in endogenous cGAMP produced by eukaryotic cGAS. ML RR-S2 CDA was shown to broadly activate all known human STING alleles in a HEK293T cellular STING signaling assay and induced dose-dependent expression of IFN- $\beta$ in human peripheral blood monocytes (PBMCs) isolated from multiple donors with different STING genotypes, including a donor homozygous for the REF allele, which is known to be refractory to signaling induced by bacterial $3^{\prime}, 3^{\prime} \mathrm{CDNs}(8,86)$.

ML RR-S2 CDA was evaluated in multiple syngeneic mouse tumor models, including B16.F10 melanoma, 4T1 mammary carcinoma, and CT26 colon carcinoma, and demonstrated a potent antitumor immune response and significant tumor regression in each model (8). Intratumoral injection of ML RR-S2 CDA three times over one week elicited both a significant reduction in mass of the treated tumor and primed a systemic $\mathrm{CD} 8^{+} \mathrm{T}$ cell response that also inhibited the growth of untreated distal flank tumors or distant lung metastases (ref. 8 and Figure 2). ML RR-S2 CDA treatment confers resistance to rechallenge with an autologous tumor, suggesting that STING CDN agonists can elicit long-term immunologic memory. Intratumoral treatment with ML RR-S2 CDA markedly increased the overall survival of B16 tumor-bearing mice. Importantly, studies conducted in STING-deficient (Goldenticket) mice (62) confirmed that the antitumor effect of ML RR-S2 CDA in mice was STING dependent (8). An additional study showing that intratumoral injection of the mammalian cGAS product cGAMP 
(formulated with Lipofectamine) induced antitumor responses in several melanoma models and in the MC38 colon carcinoma model lends further support to an approach using intratumoraladministered CDN-based STING agonists in the clinic (39).

Strategies to further enhance STING immunotherapy by combining with other immunomodulatory agents are being explored in mouse models. PD-L1 expression is increased in the tumor microenvironment of tumor-bearing mice treated with $\mathrm{CDN}$ formulated with the cancer vaccine GVAX (STINGVAX), and the combination STINGVAX and anti-PD1 was more efficacious than either treatment alone (86). These data support the notion that strategies to counteract adaptive immune resistance may enhance the potency of STING agonists in the clinic. In another study, the antitumor efficacy of cGAMP administered by intratumoral injection into B16.F10 tumors was enhanced when combined with anti-PD1 and anti-CTLA4 antibodies (39). Similarly, antitumor efficacy mediated by intratumoral injection of ML RR-S2 CDA was improved when combined with immune checkpoint inhibitors, suggesting a possible combination for future clinical development (our unpublished data). An enhancement of antitumor efficacy using STING agonists combined with a chemotherapeutic agent was reported using daily i.v. injections of mammalian cGAMP and 5-fluorouracil in the mouse CT26 colon carcinoma model (88). Combined radiotherapy and intratumorally administered CDN also worked synergistically to induce antitumor immune responses in the MC38 colon carcinoma model and PancO2 pancreatic cancer model via a mechanism involving enhancement of tumorspecific $\mathrm{CD}^{+} \mathrm{T}$ cell immunity $(73,83)$. It was also demonstrated that treatment with anti-TNF- $\alpha$ blocked CDN/radiotherapy-mediated hemorrhagic necrosis and early tumor regression, highlighting the importance of both local inflammation for the initial tumor regression and systemic immunity to induce antitumor responses (83). In addition to combination strategies to enhance the therapeutic efficacy of a STING agonist, it will be worthwhile to explore strategies for utilizing formulations of CDNs for systemic delivery to broaden the range of treatable cancer types beyond those that are easily accessible for intratumoral injection.

Clinical trials evaluating the safety and efficacy of intratumoral injection of TLR agonists into the tumor mass with innate immune modulators are completed or ongoing (89-91). Intratumoral injection of $\mathrm{CpG}$-rich oligodeoxynucleotides (CpG ODN, $\mathrm{PF}-3512676)$ in combination with low-dose radiotherapy was administered to 15 subjects with advanced non-Hodgkin's lymphoma in a phase I/II clinical study (89). This treatment was generally well tolerated and resulted in significant reductions of both treated and distal lesions in several subjects, with 13 of 15 subjects showing clinical responses. Several other TLR ligands are currently being evaluated clinically, including the TRL3/ RIG-I agonist polyinosinic-polycytidylic acid-LC (polyI:C-LC) (Hiltonol), the TLR4 agonist glucopyranosyl lipid adjuvant (GLA), and the TLR8 agonist, VTX-2337. A list of these active clinical trials is provided in Table 1.
Multiple clinical trials have also been conducted with oncolytic viruses, which may function in part through innate immune activation. Talimogene laherparepvec $(\mathrm{T}-\mathrm{Vec})$ is a recombinant engineered herpes simplex virus-1 (HSV-1) - a double-stranded DNA virus encoding granulocyte-macrophage CSF (GM-CSF) that, when given by intratumoral injection, has been shown to reduce the size of both injected and noninjected tumors. These reductions correlated with increased survival of patients with advanced melanoma (92). Interestingly, STING was found to be the requisite sensor for the induction of IFN- $\beta$ in response to HSV-1 infection (42), raising the possibility that the efficacy of T-Vec may be partially STING dependent. Clinical evaluation of these diverse innate immune modulators and encouraging preclinical efficacy with STING agonists alone and in combination with other immunomodulatory agents demonstrate the high translational potential for STING pathway engagement. Toward this objective, a Phase 1 clinical study to evaluate the safety, tolerability, and possible antitumor effects of repeated intratumorally administered doses of ML RR-S2 CDA (ADU-S100) in subjects with cutaneously accessible solid tumors and lymphomas is underway (clinicaltrials.gov, NCT02675439).

\section{Conclusions and future perspectives}

Activation of the STING pathway is a central innate immune sensing mechanism that leads to type I IFN production in the tumor microenvironment. As tumors with a type I IFN signature correlate with infiltration of $\mathrm{CD}^{+} \mathrm{T}$ cells, the use of intratumoral STING agonists holds promise as a cancer therapeutic. However, there are some questions that remain unanswered, and a deeper biologic understanding of the pathway is still needed. Mechanistically, it is still unknown how DNA is transferred to APCs from dying tumor cells in order to activate the STING pathway. In addition, the full spectrum of cell types within the tumor microenvironment that could activate the STING pathway is incompletely understood. Identification of the cell subsets that lead to type I IFN production and the functional contribution of these cells should be explored. This question is also relevant for pharmaceutical activation of the pathway, as it is possible that additional cell types activate the STING pathway and contribute to therapeutic effects. Completion of early phase clinical trials with human STING agonists will identify biologic and therapeutic effects in patients, which could also lead to combination clinical trials with checkpoint inhibitors.

\section{Acknowledgments}

L. Corrales is supported by a Cancer Research Institute Irvington postdoctoral fellowship. Some of the research discussed in this review was supported by R01 CA181160 from the National Cancer Institute.

Address correspondence to: Thomas F. Gajewski, University of Chicago, 5841 S. Maryland Ave., MC2115, Chicago, Illinois 60617, USA. Phone: 773.702.4601; E-mail: tgajewsk@medicine. bsd.uchicago.edu.
1. Kawai T, Akira S. Toll-like receptors and their crosstalk with other innate receptors in infection and immunity. Immunity. 2011;34(5):637-650.

2. Osorio F, Reis e Sousa C. Myeloid C-type lectin receptors in pathogen recognition and host defense. Immunity. 2011;34(5):651-664.

3. Elinav E, Strowig T, Henao-Mejia J, Flavell RA. Regulation of the antimicrobial response by NLR proteins. Immunity. 2011;34(5):665-679.

4. Loo YM, Gale M Jr. Immune signaling by RIG-Ilike receptors. Immunity. 2011;34(5):680-692.

5. Barber GN. STING-dependent cytosolic 
DNA sensing pathways. Trends Immunol. 2014;35(2):88-93.

6. Fuertes MB, et al. Host type I IFN signals are required for antitumor $\mathrm{CD}^{+} \mathrm{T}$ cell responses through $\mathrm{CD} 8 \alpha^{+}$dendritic cells. J Exp Med. 2011;208(10):2005-2016.

7. Woo SR, et al. STING-dependent cytosolic DNA sensing mediates innate immune recognition of immunogenic tumors. Immunity. 2014;41(5):830-842.

8. Corrales L, et al. Direct Activation of STING in the tumor microenvironment leads to potent and systemic tumor regression and immunity. Cell Rep. 2015;11(7):1018-1030.

9. Gajewski TF, Louahed J, Brichard VG. Gene signature in melanoma associated with clinical activity: a potential clue to unlock cancer immunotherapy. Cancer J. 2010;16(4):399-403.

10. Harlin $\mathrm{H}$, et al. Chemokine expression in melanoma metastases associated with $\mathrm{CD}^{+} \mathrm{T}$-cell recruitment. Cancer Res. 2009;69(7):3077-3085.

11. Ulloa-Montoya F, et al. Predictive gene signature in MAGE-A3 antigen-specific cancer immunotherapy. J Clin Oncol. 2013;31(19):2388-2395.

12. Erdag G, et al. Immunotype and immunohistologic characteristics of tumor-infiltrating immune cells are associated with clinical outcome in metastatic melanoma. Cancer Res. 2012;72(5):1070-1080.

13. Joseph RW, et al. Characterizing the clinical benefit of ipilimumab in patients who progressed on high-dose IL-2. J Immunother. 2012;35(9):711-715.

14. Hamid O, et al. A prospective phase II trial exploring the association between tumor microenvironment biomarkers and clinical activity of ipilimumab in advanced melanoma. J Transl Med. 2011;9:204.

15. Ji RR, et al. An immune-active tumor microenvironment favors clinical response to ipilimumab. Cancer Immunol Immunother. 2012;61(7):1019-1031.

16. Topalian SL, et al. Safety, activity, and immune correlates of anti-PD-1 antibody in cancer. $N$ Engl JMed. 2012;366(26):2443-2454.

17. Spranger S, Koblish HK, Horton B, Scherle PA, Newton R, Gajewski TF. Mechanism of tumor rejection with doublets of CTLA-4, PD-1/PD-L1, or IDO blockade involves restored IL-2 production and proliferation of CD8(+) T cells directly within the tumor microenvironment. JImmunother Cancer. 2014;2:3.

18. Tumeh PC, et al. PD-1 blockade induces responses by inhibiting adaptive immune resistance. Nature. 2014;515(7528):568-571.

19. Azimi F, et al. Tumor-infiltrating lymphocyte grade is an independent predictor of sentinel lymph node status and survival in patients with cutaneous melanoma. JClin Oncol. 2012;30(21):2678-2683.

20. Galon J, et al. Type, density, and location of immune cells within human colorectal tumors predict clinical outcome. Science. 2006;313(5795):1960-1964.

21. Mahmoud SM, et al. Tumor-infiltrating CD8 ${ }^{+}$ lymphocytes predict clinical outcome in breast cancer. JClin Oncol. 2011;29(15):1949-1955.

22. Rusakiewicz S, et al. Immune infiltrates are prognostic factors in localized gastrointestinal stromal tumors. Cancer Res. 2013;73(12):3499-3510.
23. Zhang L, et al. Intratumoral T cells, recurrence, and survival in epithelial ovarian cancer. $N$ Engl J Med. 2003;348(3):203-213.

24. Valmori D, Lienard D, Waanders G, Rimoldi D, Cerottini JC, Romero P. Analysis of MAGE-3-specific cytolytic T lymphocytes in human leukocyte antigen-A2 melanoma patients. Cancer Res. 1997;57(4):735-741.

25. Robbins PF, et al. Mining exomic sequencing data to identify mutated antigens recognized by adoptively transferred tumor-reactive T cells. Nat Med. 2013;19(6):747-752.

26. Rizvi NA, et al. Cancer immunology. Science. 2015;348(6230):124-128.

27. Sistigu A, et al. Cancer cell-autonomous contribution of type I interferon signaling to the efficacy of chemotherapy. Nat Med. 2014;20(11):1301-1309.

28. Zitvogel L, Galluzzi L, Kepp O, Smyth MJ, Kroemer G. Type I interferons in anticancer immunity. Nat Rev Immunol. 2015;15(7):405-414.

29. Dunn GP, et al. A critical function for type I interferons in cancer immunoediting. Nat Immunol. 2005;6(7):722-729.

30. Diamond MS, et al. Type I interferon is selectively required by dendritic cells for immune rejection of tumors. J Exp Med. 2011;208(10):1989-2003.

31. Hildner K, et al. Batf3 deficiency reveals a critical role for $\mathrm{CD} 8 \alpha^{+}$dendritic cells in cytotoxic $\mathrm{T}$ cell immunity. Science. 2008;322(5904):1097-1100.

32. Bidwell BN, et al. Silencing of Irf7 pathways in breast cancer cells promotes bone metastasis through immune escape. Nat Med. 2012;18(8):1224-1231.

33. Savitsky D, Tamura T, Yanai H, Taniguchi T. Regulation of immunity and oncogenesis by the IRF transcription factor family. Cancer Immunol Immunother. 2010;59(4):489-510.

34. Stetson DB, Ko JS, Heidmann T, Medzhitov R. Trex1 prevents cell-intrinsic initiation of autoimmunity. Cell. 2008;134(4):587-598.

35. Lau L, Gray EE, Brunette RL, Stetson DB. DNA tumor virus oncogenes antagonize the cGAS-STING DNA-sensing pathway. Science. 2015;350(6260):568-571.

36. Moore PS, Chang Y. Why do viruses cause cancer? Highlights of the first century of human tumour virology. Nat Rev Cancer. 2010;10(12):878-889.

37. Diaz MO, et al. Homozygous deletion of the $\alpha$ - and $\beta 1$-interferon genes in human leukemia and derived cell lines. Proc Natl Acad Sci U S A. 1988;85(14):5259-5263.

38. Olopade OI, et al. Molecular analysis of deletions of the short arm of chromosome 9 in human gliomas. Cancer Res. 1992;52(9):2523-2529.

39. Demaria O, et al. STING activation of tumor endothelial cells initiates spontaneous and therapeutic antitumor immunity. Proc Natl Acad Sci U S A. 2015;112(50):15408-15413.

40. Ahn J, Gutman D, Saijo S, Barber GN. STING manifests self DNA-dependent inflammatory disease. Proc Natl Acad Sci U S A. 2012;109(47):19386-19391.

41. Rongvaux A, et al. Apoptotic caspases prevent the induction of type I interferons by mitochondrial DNA. Cell. 2014;159(7):1563-1577.

42. Ishikawa $\mathrm{H}, \mathrm{Ma} Z$, Barber GN. STING regulates intracellular DNA-mediated, type I inter- feron-dependent innate immunity. Nature. 2009;461(7265):788-792.

43. Liu S, et al. Phosphorylation of innate immune adaptor proteins MAVS, STING, and TRIF induces IRF3 activation. Science. 2015;347(6227):aaa2630.

44. Burdette DL, et al. STING is a direct innate immune sensor of cyclic di-GMP. Nature. 2011;478(7370):515-518.

45. Burdette DL, Vance RE. STING and the innate immune response to nucleic acids in the cytosol. Nat Immunol. 2013;14(1):19-26.

46. Ishikawa H, Barber GN. STING is an endoplasmic reticulum adaptor that facilitates innate immune signalling. Nature. 2008;455(7213):674-678.

47. Dempsey A, Bowie AG. Innate immune recognition of DNA: A recent history. Virology. 2015;479-480:146-152.

48. Romling U, Galperin MY, Gomelsky M. Cyclic di-GMP: the first 25 years of a universal bacterial second messenger. Microbiol Mol Biol Rev. 2013;77(1):1-52.

49. McWhirter SM, et al. A host type I interferon response is induced by cytosolic sensing of the bacterial second messenger cyclic-di-GMP. J Exp Med. 2009;206(9):1899-1911.

50. Wu J, et al. Cyclic GMP-AMP is an endogenous second messenger in innate immune signaling by cytosolic DNA. Science. 2013;339(6121):826-830.

51. Diner EJ, et al. The innate immune DNA sensor cGAS produces a noncanonical cyclic dinucleotide that activates human STING. Cell Rep. 2013;3(5):1355-1361.

52. Gao P, et al. Cyclic $\left[G\left(2^{\prime}, 5^{\prime}\right) \mathrm{pA}\left(3^{\prime}, 5^{\prime}\right) \mathrm{p}\right]$ is the metazoan second messenger produced by DNA-activated cyclic GMP-AMP synthase. Cell. 2013;153(5):1094-1107.

53. Ablasser A, et al. cGAS produces a 2'-5'-linked cyclic dinucleotide second messenger that activates STING. Nature. 2013;498(7454):380-384.

54. Sun L, Wu J, Du F, Chen X, Chen ZJ. Cyclic GMPAMP synthase is a cytosolic DNA sensor that activates the type I interferon pathway. Science. 2013;339(6121):786-791.

55. Li XD, Wu J, Gao D, Wang H, Sun L, Chen ZJ. Pivotal roles of cGAS-cGAMP signaling in antiviral defense and immune adjuvant effects. Science. 2013;341(6152):1390-1394.

56. Molinero LL, et al. Epidermal Langerhans cells promote skin allograft rejection in mice with NF-кB-impaired T cells. Am J Transplant. 2008;8(1):21-31.

57. Ahn J, Konno H, Barber GN. Diverse roles of STING-dependent signaling on the development of cancer. Oncogene. 2015;34(41):5302-5308.

58. Zhu Q, et al. Cutting edge: STING mediates protection against colorectal tumorigenesis by governing the magnitude of intestinal inflammation. JImmunol. 2014;193(10):4779-4782.

59. Huber S, et al. IL-22BP is regulated by the inflammasome and modulates tumorigenesis in the intestine. Nature. 2012;491(7423):259-263.

60. Salcedo R, et al. MyD88-mediated signaling prevents development of adenocarcinomas of the colon: role of interleukin 18. J Exp Med. 2010;207(8):1625-1636.

61. Yu H, Pardoll D, Jove R. STATs in cancer inflammation and immunity: a leading role for STAT3. 
Nat Rev Cancer. 2009;9(11):798-809.

62. Sauer JD, et al. The N-ethyl-N-nitrosoureainduced Goldenticket mouse mutant reveals an essential function of Sting in the in vivo interferon response to Listeria monocytogenes and cyclic dinucleotides. Infect Immun. 2011;79(2):688-694.

63. Ohkuri T, et al. STING contributes to antiglioma immunity via triggering type I IFN signals in the tumor microenvironment. Cancer Immunol Res. 2014;2(12):1199-1208

64. Klarquist J, Hennies CM, Lehn MA, Reboulet RA, Feau S, Janssen EM. STING-mediated DNA sensing promotes antitumor and autoimmune responses to dying cells. JImmunol. 2014;193(12):6124-6134.

65. Ahn J, Xia T, Konno H, Konno K, Ruiz P, Barber GN. Inflammation-driven carcinogenesis is mediated through STING. Nat Commun. 2014;5:5166.

66. Barber GN. STING: infection, inflammation and cancer. Nat Rev Immunol. 2015;15(12):760-770.

67. Lemos H, Huang L, McGaha TL, Mellor AL. Cytosolic DNA sensing via the stimulator of interferon genes adaptor: Yin and Yang of immune responses to DNA. Eur J Immunol. 2014;44(10):2847-2853.

68. Huang L, et al. Cutting edge: DNA sensing via the STING adaptor in myeloid dendritic cells induces potent tolerogenic responses. JImmunol. 2013;191(7):3509-3513.

69. Lemos $\mathrm{H}$, et al. STING promotes the growth of tumors characterized by low antigenicity via IDO activation. Cancer Res. 2016;76(8):2076-2081.

70. Poltorak A, Larkin B, Surpris G, Ilyuha V. The role of STING in T lymphocytes (IRM5P). J Immunol. 2015;194(1 suppl):59.8.

71. Xia T, Konno H, Ahn J, Barber GN. Deregulation of STING signaling in colorectal carcinoma donstrains DNA damage responses and correlates with tumorigenesis. Cell Rep. 2016;14(2):282-297.

72. Hartlova A, et al. DNA damage primes the type I interferon system via the cytosolic DNA sensor
STING to promote anti-microbial innate immunity. Immunity. 2015;42(2):332-343.

73. Deng L, et al. STING-dependent cytosolic DNA sensing promotes radiation-induced type I interferon-dependent antitumor immunity in immunogenic tumors. Immunity. 2014;41(5):843-852.

74. Baguley BC, Ching LM. Immunomodulatory actions of xanthenone anticancer agents. BioDrugs. 1997;8(2):119-127.

75. Conlon J, et al. Mouse, but not human STING, binds and signals in response to the vascular disrupting agent 5,6-dimethylxanthenone-4-acetic acid. J Immunol. 2013;190(10):5216-5225.

76. Gao P, et al. Structure-function analysis of STING activation by $\mathrm{c}\left[\mathrm{G}\left(2^{\prime}, 5^{\prime}\right) \mathrm{pA}\left(3^{\prime}, 5^{\prime}\right) \mathrm{p}\right]$ and targeting by antiviral DMXAA. Cell. 2013;154(4):748-762.

77. Kim S, Li L, Maliga Z, Yin Q, Wu H, Mitchison TJ. Anticancer flavonoids are mouse-selective STING agonists. ACS Chem Biol. 2013;8(7):1396-1401.

78. Prantner D, et al. 5,6-Dimethylxanthenone4-acetic acid (DMXAA) activates stimulator of interferon gene (STING)-dependent innate immune pathways and is regulated by mitochondrial membrane potential. J Biol Chem. 2012;287(47):39776-39788.

79. Ching LM, Cao Z, Kieda C, Zwain S, Jameson $\mathrm{MB}$, Baguley BC. Induction of endothelial cell apoptosis by the antivascular agent 5,6-Dimethylxanthenone-4-acetic acid. BrJCancer. 2002;86(12):1937-1942.

80. Moilanen E, Thomsen LL, Miles DW, Happerfield DW, Knowles RG, Moncada S. Persistent induction of nitric oxide synthase in tumours from mice treated with the anti-tumour agent 5,6-dimethylxanthenone-4-acetic acid. Br J Cancer. 1998;77(3):426-433.

81. Sun J, et al. Activation of mitogen-activated protein kinases by 5,6-dimethylxanthenone-4-acetic acid (DMXAA) plays an important role in macrophage stimulation. Biochem Pharmacol. 2011;82(9):1175-1185.

82. Downey CM, Aghaei M, Schwendener RA, Jirik FR. DMXAA causes tumor site-specific vascular disruption in murine non-small cell lung cancer, and like the endogenous non-canonical cyclic dinucleotide STING agonist, 2'3'-cGAMP, induces M2 macrophage repolarization. PLoS One. 2014;9(6):e99988.

83. Baird JR, et al. Radiotherapy Combined with novel STING-targeting oligonucleotides results in regression of established tumors. Cancer Res. 2016;76(1):50-61.

84. Lara PN. Randomized phase III placebo-controlled trial of carboplatin and paclitaxel with or without the vascular disrupting agent vadimezan (ASA404) in advanced non-small-cell lung cancer. JClin Oncol. 2011;29(22):2965-2971.

85. Chandra D, et al. STING ligand c-di-GMP improves cancer vaccination against metastatic breast cancer. Cancer Immunol Res. 2014;2(9):901-910.

86. Fu J, et al. STING agonist formulated cancer vaccines can cure established tumors resistant to PD-1 blockade. Sci Transl Med. 2015;7(283):283ra252.

87. Yi G, Brendel VP, Shu C, Li P, Palanathan S, Cheng Kao C. Single nucleotide polymorphisms of human STING can affect innate immune response to cyclic dinucleotides. PLoS One. 2013;8(10):e77846.

88. Li T, et al. Antitumor activity of cGAMP via stimulation of cGAS-cGAMP-STING-IRF3 mediated innate immune response. Sci Rep. 2016;6:19049.

89. Brody JD, et al. In situ vaccination with a TLR9 agonist induces systemic lymphoma regression: a phase I/II study.JClin Oncol.2010;28(28):4324-4332.

90. Caskey M, et al. Synthetic double-stranded RNA induces innate immune responses similar to a live viral vaccine in humans. J Exp Med. 2011;208(12):2357-2366.

91. Dowling DJ, et al. The ultra-potent and selective TLR8 agonist VTX-294 activates human newborn and adult leukocytes. PLoS One. 2013;8(3):e58164.

92. Harrington KJ, et al. Clinical development of talimogene laherparepvec (T-VEC): a modified herpes simplex virus type-1-derived oncolytic immunotherapy. Expert Rev Anticancer Ther. 2015;15(12):1389-1403. 